\title{
Evolution of Cooperation in a Population of Selfish Adaptive Agents
}

\author{
Jorge M. Pacheco ${ }^{1}$, Tom Lenaerts ${ }^{2,3}$, and Francisco C. Santos ${ }^{4}$ \\ 1 Centro de Física Teórica e Computacional and Departamento de Física da \\ Faculdade de Ciências da Universidade de Lisboa, Lisbon, Portugal ** \\ 2 SWITCH Laboratory, VIB, Gent, Belgium \\ 3 SWIT, Vrije Universiteit Brussel, Brussels, Belgium \\ ${ }^{4}$ IRIDIA, CoDE, Université Libre de Bruxelles, Brussels, Belgium
}

\begin{abstract}
Often the selfish and strong are believed to be favored by natural selection, even though cooperative interactions thrive at all levels of organization in living systems. Recent empirical data shows that networks representing the social interactions between people exhibit typically high average connectivity and associated single-to-broad-scale heterogeneity, a feature which precludes the emergence of cooperation in any static network. Here, we employ a model in which individuals are able to self-organize both their strategy and their social ties throughout evolution, based exclusively on their self-interest. The entangled evolution of individual strategy and network structure provides a key mechanism toward the sustainability of cooperation in social networks. The results show that simple topological dynamics reflecting the individual capacity for self-organization of social ties can produce realistic networks of high average connectivity with associated single-to-broad-scale heterogeneity, in which cooperation thrives.
\end{abstract}

\section{Introduction}

Conventional evolutionary game theory predicts that natural selection favors the selfish and strong [1], despite existing evidence showing that cooperation is more widespread than theory predicts [2].

When cooperation is modeled in terms of the prisoner's dilemma [3] (PD), the solution of the replicator dynamics equation in infinite, well-mixed populations 41516] dictates the extinction of cooperators. Cooperation becomes evolutionarily competitive whenever individuals are constrained to interact with few others along the links of sparse graphs, as initially predicted in Ref. 7] and more recently concluded in two independent studies which extend the original work to general social dilemmas described in terms of symmetric two-player games [8]9. Both studies place individuals on the nodes of a static graph, and associate their social ties with the edges linking the nodes such that, throughout evolution, every individual has the possibility of changing her strategy, but not her social

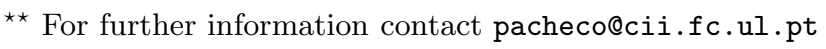


ties. In both studies the authors concluded that games on graphs open a window for the emergence of cooperation, inasmuch as graphs are sparse, that is, the average connectivity $z$ (see section 3), is small.

However, recent data shows that realistic networks [101112 13 14 15] exhibit values of $z$ ranging from 2 to 170 (see methods), with an associated heterogeneity intermediate between single and broad-scale [11, which differs from the connectivity values typically used in previous studies 89. Indeed, whereas single scale networks are characterized by a degree distribution peaked around a well defined value, such that most nodes exhibit a similar average connectivity pattern (homogeneity), broad scale networks are characterized by a more heterogeneous degree distribution, with different nodes exhibiting markedly different connectivities (cf. Fig. 4).

For instance, the network of movie actors exhibits an average connectivity of 30 [16], whereas collaboration networks based on co-authorship of published papers vary from average values of 4 (mathematics) up to 15 (biology) [13. None of the previous results is capable of explaining how cooperation thrives on such social networks. Other mechanisms have to be at work here that allow for the survival of cooperation.

In most evolutionary models developed so far, social interactions are fixed from the outset. Such immutable social ties, associated naturally with static graphs, imply that individuals have no control over the number, frequency, or duration of their ties; they can only evolve their behavioral strategy. A similar observation can be made on studies related to the physical properties of complex networks [10 11 12 13 14 15]. The analyzed networks constitute but one static snapshot of networks that have been typically produced by some growth process.

Yet, networks have naturally evolved before and will continue to evolve after the snapshot has been taken. Indeed, recent longitudinal studies of evolving social networks 17/18 indicate that global properties seem to remain rather stable, whereas individual patterns of social ties keep evolving in time. Here, we employ a minimal model that combines strategy evolution with topological evolution, in which the requirements of individual cognitive capacities are very small, and investigate under which conditions cooperation may thrive. Network heterogeneity, which now emerges as a result of an entangled co-evolutionary dynamics, will be shown to play a crucial role in facilitating cooperative behavior.

\section{Entangled Evolution of Strategy and Structure}

\subsection{The Prisoner's Dilemma}

We consider two types of individuals-cooperators and defectors, who engage the most popular social dilemma of cooperation : The Prisoner's Dilemma $(P D)$ (see below). They are not required to accumulate information on all other players, only those they are immediately connected with. The $P D$ is modeled as a symmetric two-player game in which the players can either cooperate or defect when they interact. When both cooperate, they receive the payoff $R$ (the reward for mutual cooperation). On the other hand, when both defect, they both obtain the 


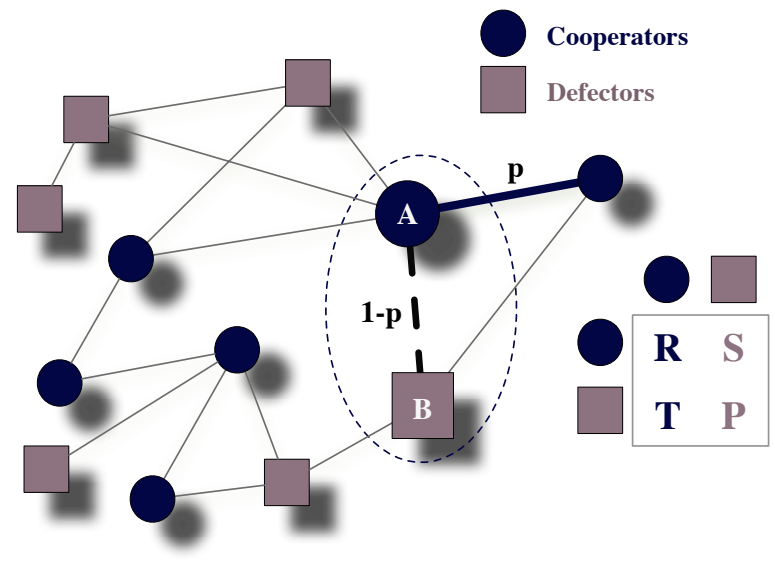

Fig. 1. Readjusting social ties. Cooperators and defectors interact via the links of a network. $B(A)$ is satisfied (dissatisfied), since $A(B)$ is a cooperator (defector). Therefore, $A$ wants to change the link whereas $B$ does not. The action taken is contingent on the fitness $\Pi(A)$ and $\Pi(B)$ of $A$ and $B$, respectively. With probability $p$ (see 3), $A$ redirects the link to a random neighbor of $B$. With probability $1-p, A$ stays linked to $B$. Other possibilities may occur, as detailed in section 3

payoff $P$ (the punishment for mutual defection). The two remaining possibilities occur when one defects and the other cooperates, resulting in the payoff $T$ (the temptation to cheat) for the defector and $S$ (the disadvantage of being cheated) for the cooperator. The dilemma follows from the players' payoff preferences. Players are referred to as greedy since they prefer unilateral defection to mutual cooperation $(T>R)$. Moreover, mutual defection is preferred to unilateral cooperation $(S<P)$, resulting in an intrinsic fear for the players to cooperate. We adopt the convention of [8] and normalize the difference between mutual cooperation $(R)$ and mutual defection $(P)$ to 1 , making $R=1$ and $P=0$, respectively. Consequently, we investigate the $P D$ in a $2 \mathrm{D}$ parameter space, depicted in Figure 2, where the payoff $T$ (temptation to cheat) satisfies $0 \leq T \leq 2$ and the payoff $S$ (disadvantage of being cheated) satisfies $-1 \leq S \leq 0$. The fitness of each individual corresponds to the total accumulated payoff resulting from pairwise interactions (see section 3 ) with all her neighbors.

\subsection{Quality Assessment of Social Ties}

Both cooperators and defectors are able to decide, on an equal footing, those ties that they want to maintain and those they want to change. Given an edge with individuals $\mathrm{A}$ and $\mathrm{B}$ at the extremes, we say that $\mathrm{A}(\mathrm{B})$ is satisfied with the edge if the strategy of $\mathrm{B}(\mathrm{A})$ is a cooperator, being dissatisfied otherwise. If $\mathrm{A}$ is satisfied, she will decide to maintain the link. If dissatisfied, then she 


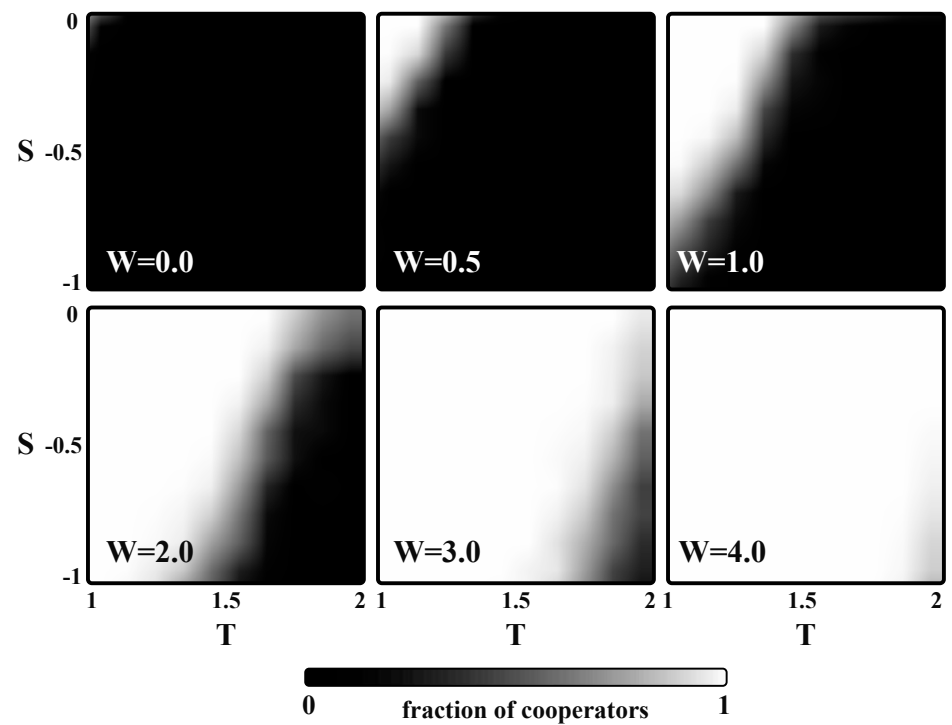

Fig. 2. Co-Evolution in the $P D$ for different time-scales. Results for the fraction of successful evolutionary runs ending in $100 \%$ cooperation for different values of the time scale ratio $W$. We study the $P D$ in the area $2 \geq T \geq 1>0 \geq S \geq-1$. For $W=0\left(N=10^{3}, z=30\right.$ and $\left.\beta=0.005\right)$, the results fit the predictions from wellmixed populations. With increasing $W$ it also increases the rate at which individuals readjust their ties, and so does the viability of cooperation. Above a critical value $W_{\text {critical }} \sim 4.0$ (see also Fig. 3), cooperators efficiently wipe out defectors. For the strategy evolution dynamics adopted here (pairwise comparison, see section 3), and according to 9 , cooperation would never be favored. The contours provide arresting evidence of the viability of cooperation under co-evolutionary dynamics.

may compete with B to rewire the link (see Fig. 1 and section 3), rewiring being attempted to a random neighbor of $\mathrm{B}$. The intuition behind this reasoning relies on the fact that agents, equipped with limited knowledge and scope, look for new social ties by proxy [17. In this sense, agent $\mathrm{A}$ is more likely to encounter one of the friends of B and become a friend with B's neighbor. Moreover, selecting a neighbor of an inconvenient partner may turn out to be a good choice, since this partner also tries to establish links with cooperators, making it more likely that the rewiring results in a tie to a cooperator. Indeed, it is always best to have a link with a cooperator, as it maximizes the fitness of any individual, irrespective of her (fixed) strategy. Consequently, all individuals naturally seek to establish links with cooperators. As a result, rewiring to a neighbor of a defector is certainly a good choice for individuals with local information only. 


\subsection{Different Evolutionary Timescales}

The fact that in our model cooperators and defectors interact via social ties they both decide upon establishes a coupling between individual strategy and population structure: the game payoff induces now an entangled co-evolution of strategy and structure. Such an adaptive individual behavior introduces a new time scale $\left(\tau_{a}\right)$, not necessarily equal to the time scale associated with strategy evolution $\left(\tau_{e}\right)$. Depending on the ratio $W=\tau_{e} / \tau_{a}$, different fates may occur for cooperation. Indeed, whenever $\tau_{e}<<\tau_{a}$, that is, $W \rightarrow 0$, we recover the results of $[89$. On the other hand, with increasing $W$, individuals become apt to adapt their ties with increasing efficiency. In general, however, one expects the two time scales to be of comparable magnitude in realistic situations (see Figs. 2 and 3 ). More intuitively, $W$ provides a measure of individuals' responsiveness to adverse ties: large values of $\mathrm{W}$ reflect populations in which individuals react promptly to adverse ties, whereas smaller values of $W$ reveal the opposite behaviour. In reality, individuals may exhibit their own characteristic $W$. We shall not dwell into these intricacies here. Instead, we view $W$ as reflecting an average feature of the population.

\section{Materials and Methods}

\subsection{Graphs}

We place individuals on the nodes (a total of $N$ ) of a graph. Links (a total of $N_{E}$ ) represent the social ties between individuals. Graphs evolve in time as individuals change their ties. The average connectivity $z=2 N_{E} / N$ is conserved since we do not introduce or destroy links. At all times graphs are required to remain connected. We enforce this condition by imposing that nodes connected by a single link cannot loose this link. We also computed the cumulative degree distribution $D(k)=N^{-1} \sum_{i=k}^{N-1} N_{i}$ (where $N_{i}$ gives the number of nodes with $i$ links), where $D(k)$ gives the probability of finding nodes in the graph with degree greater or equal to $k$. Since $D(k)=0$ for $k>k_{\max }$, with $k_{\max }$ the maximum value of the connectivity of a graph, both $h$ and $k_{\max }$ provide simple measures of the heterogeneity of a graph.

\subsection{Evolution of Strategies}

Whenever $W>0$, evolution of strategy and structure proceed together under asynchronous updating. Choice of type of update event depends on $W$; assuming (without loss of generality) $\tau_{e}=1$, a strategy update event is chosen with probability $(1+W)^{-1}$, a structural update event being selected otherwise. A strategy update event is defined in the following way, corresponding to the so-called pairwise comparison rule [24]: One node $A$ is chosen at random and another node $B$ is chosen randomly among $A$ 's first neighbors. The individuals $A$ and $B$ interact with all their neighbors (those directly connected to them by links) according to $P D$. As a result, they accumulate total payoffs $\Pi(A)$ and $\Pi(B)$, respectively. 


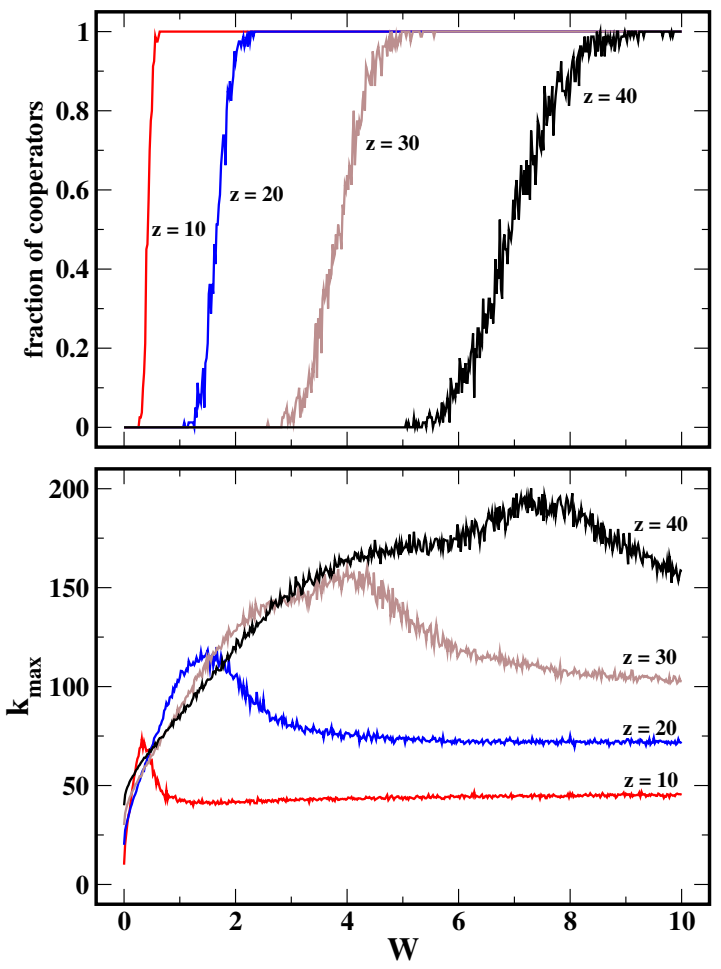

Fig. 3. Co-Evolution in the $P D$ for different time-scales. $P D$ with $T=2, S=$ -1 and $\beta=0.005$. Upper panel Fraction of cooperators at end as a function of $W$ for different values of $z$. For each value of $z$, there is a critical value of $W-W_{\text {critical }}$ - above which cooperators wipe out defectors. Lower panel Maximum value of the connectivity in population as a function of $W$. With increasing $z, W_{\text {critical }}$ increases. In all cases, the heterogeneity of the associated network becomes maximal at $W_{\text {critical }}$, stagnating for higher values.

The strategy of $B$ replaces that of $A$ with a probability given by the (Fermi distribution) function $p=\left[1+e^{-\beta[\Pi(B)-\Pi(A)]}\right]^{-1}$. The value of $\beta \geq 0$, which plays the role of an inverse temperature in statistical physics, controls here the intensity of selection [24] $\beta \rightarrow 0$ leads to neutral drift whereas $\beta \rightarrow \infty$ leads to the so-called imitation dynamics, often used to model cultural evolution.

\subsection{Link Rewiring}

Cooperators and defectors interact via the links of a network. Two individuals, $A$ and $B$, connected by one link, may be satisfied or dissatisfied, as shown in Fig. 1 . In Fig. 1, $B$ is satisfied, whereas $A$ is not, since $A(B)$ is a cooperator (defector). Therefore, $A$ wants to change the link whereas $B$ does not. The action taken is 
contingent on the fitness $\Pi(A)$ and $\Pi(B)$ of $A$ and $B$, respectively. With the probability $p$ defined above in terms of the Fermi distribution, $A$ redirects the link to a random neighbor of $B$. With probability $1-p, A$ stays linked to $B$. Whenever both $A$ and $B$ are satisfied, nothing happens. When both $A$ and $B$ are dissatisfied, rewiring takes place such that the new link keeps attached to $A$ with probability $p$ and attached to $B$ with probability $1-p$.

\subsection{Computer Simulations}

We start from a homogeneous random graph [25], in which all nodes have the same number of links $(z)$, randomly linked to arbitrary nodes. Population size is $N=10^{3}$ and average connectivities $z=10,20,30$, and 40 (the value $z=30$ used in Fig. 2 reflects the mean value of the average connectivities reported in 13 . for socials networks). We start with $50 \%$ of cooperators randomly distributed in the population. We run 100 independent simulations for each set of parameters $(T, S, W)$ and compute the fraction of times that evolution stopped at $100 \%$ cooperation. Indeed, under stochastic dynamics the population will invetitably converge to one of the two possible absorbing states: $100 \%$ cooperators or $100 \%$ defectors; consequently, each run proceeds for as many iterations as necessary until one of these absorbing states is reached. At the end of each evolution we also computed the maximal connectivity $k_{\max }$ associated with the final graph and the cumulative degree distribution, which are on the basis of the results plotted in Figures 3 and 4 . We have confirmed that our results are valid for $N>500$.

\section{Results and Discussion}

The contour plots in Fig. 2 show how cooperators survive for different values of the ratio $W$ in networks with high connectivity. We plot the fraction of cooperators who survive evolution, averaged over 100 independent realizations for the same values of the temptation to cheat $T$, the disadvantage of being cheated $S$, and $W$. For $W=0$ the results reproduce, as expected [8], the predictions for finite, well-mixed populations 819]. Yet, with increasing $W$ we observe a wave of cooperation moving southeast toward the lower right corner of each contour plot. Hence, the $P D$ for $T=2$ and $S=-1$ constitutes the hardest challenge for cooperation within the parameter space studied. Nonetheless, for sufficiently large values of $W$, one obtains as a result of the entangled co-evolution of strategy and structure a full cooperative scenario: The swifter the response of individuals to the nature of their ties, the easier it gets for cooperators to wipe out defectors. Additional insight is provided in Fig. 3 (upper panel), where we investigate how cooperation wins over defection as a function of $W$ for the $P D$ when $T=2$ and $S=-1$ and different values of the average connectivity $z$. For small $W$, cooperators have no chance. Their fate changes as $W$ approaches a critical value $W_{\text {critical }}$ - which increases monotonically with $z$ - cooperators wiping out defectors above $W_{\text {critical }}$ (the increase of $W_{\text {critical }}$ with $z$ is expected, since there 
are more links to be rewired; in practice, $W_{\text {critical }}$ is determined as the value of $W$ at which cooperation reaches $50 \%$ ). Thus, the survival of cooperation relies on the capacity of individuals to adjust to adverse ties, even when the average connectivity is high. Figure 3 also provides evidence of the detailed interplay between strategy and structure. On one hand, strategy updating promotes a local assortment of strategies, since cooperators breed cooperators and defectors breed defectors. On (static) heterogeneous graphs, and for specific values of $\mathrm{T}$ and $\mathrm{S}$, the population will evolve into a cooperative state [8, and the transient dynamics associated with the approach to such a cooperative state, starting from an equal fraction of cooperators and defectors, has been examined in detail in 720 . On the other hand, under structural updating, however, one is promoting local assortative interactions between cooperators (that is, cooperator-cooperator links) and disassortative interactions between defectors and cooperators (that is, cooperator-defector links), which constitute favorable steps from an individual point of view. Clearly, when simultaneously active, strategy update will reinforce assortativity among cooperators, but will inhibit disassortativity between defectors and cooperators, which overall will benefit the emergence of cooperation. Furthermore, since for any finite $\mathrm{W}$ graph heterogeneity will develop as a result of structural update (we are starting from homogeneous graphs), it will become easier for strategy update to promote cooperation.

For any $W>0$, individual choices lead to heterogeneous graphs in which some individuals interact more, and more often than, others. The overall onset of increase of heterogeneity qualitatively follows the wave of cooperation shown in Fig. 21 21. In fact, the overall heterogeneity of the graph increases as $W$ increases reaching a maximum at $W_{\text {critical }}$, above which heterogeneity decreases again down to a stationary value 21]. This is clearly shown in Fig. 4 for the case $T=$ $2, S=-1$. The results shown suggest that the adaptive dynamics of social ties introduced here coupled with social dilemmas accounts for the heterogeneities observed in realistic social networks [11. Similar (analytic) results were obtained recently in a simpler model of link rewiring [22 23].

To conclude, the results presented in this manuscript show that to understand the emergence of cooperative behavior in a realistic scenario, one should consider simultaneously the evolution of the social network of interactions and the evolution of individual strategies. Besides providing a bottom-up answer to the conundrum of cooperation, the proposed mechanism also shows how complex social topologies can result from simple social dynamical processes, exclusively based in local assumptions.

Acknowledgments. Discussions with Martin A. Nowak, Arne Traulsen, Sebastian Maurer-Stroh, Hisashi Ohtsuki and Hugues Bersini are gratefully acknowledged. JMP acknowledges support from FCT, Portugal. FCS acknowledges the support of $\mathrm{COMP}^{2} \mathrm{SYS}$, a Marie Curie Early Stage Training Site, funded by the EC through the HRM activity. 


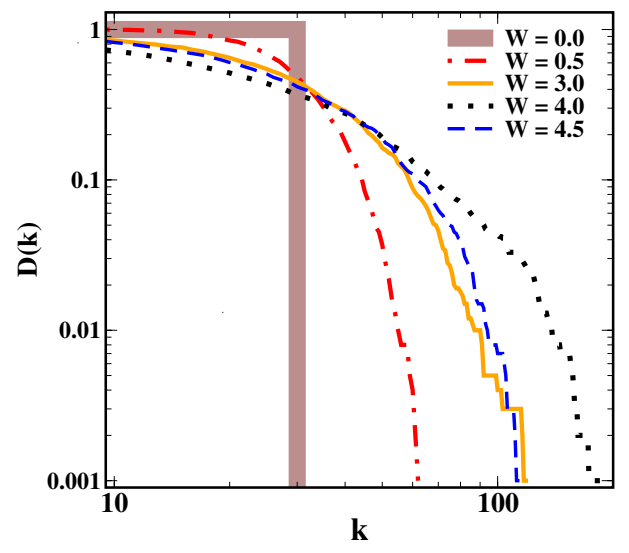

Fig. 4. Evolution of heterogeneity. Cumulative degree distributions (see section 3) for $P D$ with $T=2$ and $S=-1$. Starting from a distribution exhibiting a sharp cutoff at $k_{\max }=z$, as soon as $W>0$, the distribution widens, resulting in both single scale networks $(W=0.5$, dash-dotted line) and broad-scale networks $(W>3$, solid and dotted lines), reflecting the increase in the overall heterogeneity of the pattern of ties in the population. For $W>W_{\text {critical }}$ heterogeneity decreases again, as with increasing $W$ it is easier for cooperators to wipe out defectors. Consequently, heterogeneity helps cooperators eradicate defectors.

\section{References}

1. Nowak, M.A., Sigmund, K.: Evolution of indirect reciprocity. Nature 437, 1291$1298(2005)$

2. Smith, J.M., Szathmáry, E.: The Major Transitions in Evolution. Freeman, Oxford (1995)

3. Rapoport, A., Chamah, A.M.: The prisoner's dilemma. University of Michigan Press, Ann Arbor (1965)

4. Hofbauer, J., Sigmund, K.: Evolutionary games and population dynamics. Cambridge University Press, Cambridge (1998)

5. Gintis, H.: Game theory evolving. Cambridge University Press, Cambridge (2000)

6. Nowak, M.A., Sigmund, K.: Evolutionary dynamics of biological games. Science 303, 793-799 (2004)

7. Santos, F.C., Pacheco, J.M.: Scale-free networks provide a unifying framework for the emergence of cooperation. Phys. Rev. Lett. 95, 098104 (2005)

8. Santos, F.C., Pacheco, J.M., Lenaerts, T.: Evolutionary dynamics of social dilemmas in structured heterogeneous populations. Proc. Natl. Acad. Sci. U S A 103, 3490-3494 (2006)

9. Ohtsuki, H., Hauert, C., Lieberman, E., Nowak, M.A.: A simple rule for evolution of cooperation on graphs and social networks. Nature 441, 502-505 (2006)

10. Watts, D.J.: Small worlds: The dynamics of networks between order and randomness. Princeton University Press, Princeton (1999)

11. Amaral, L.A., Scala, A., Barthelemy, M., Stanley, H.E.: Classes of small-world networks. Proc. Natl. Acad. Sci. U S A 97, 11149-11152 (2000) 
12. Albert, R., Barabási, A.L.: Statistical mechanics of complex networks. Rev. Mod. Phys. 74, 47-98 (2002)

13. Dorogotsev, S., Mendes, J.: Evolution of networks: From biological nets to the Internet and WWW. Oxford University Press, Oxford (2003)

14. Watts, D.J.: The "new" science of networks. Ann. Rev. Sociobiol. 30, 243-270 (2004)

15. Guimerá, R., Amaral, L.A.N.: Functional cartography of complex metabolic networks. Nature 433, 895 (2005)

16. Albert, R., Barabasi, A.L.: Topology of evolving networks: Local events and universality. Phys. Rev. Lett. 85, 5234-5237 (2000)

17. Kossinets, G., Watts, D.J.: Empirical analysis of an evolving social network. Phys. Rev. Lett. 311, 89-90 (2006)

18. Watts, D.J.: A new science for the 21st century. Nature 445, 489 (2007)

19. Santos, F.C., Rodrigues, J.F., Pacheco, J.M.: Graph topology plays a determinant role in the evolution of cooperation. Proc. Biol. Sci. 273, 51-55 (2006)

20. Santos, F.C., Pacheco, J.M.: A new route to the evolution of cooperation. J. Evol. Biol. 19, 726-733 (2006)

21. Santos, F.C., Pacheco, J.M., Lenaerts, T.: Cooperation prevails when individuals adjust their social ties. PLoS Computational Biology 2, 1284 (2006)

22. Pacheco, J.M., Traulsen, A., Nowak, M.A.: Coevolution of strategy and structure in complex networks with dynamical linking. Phys. Rev. Lett. 97, 258103 (2006)

23. Pacheco, J.M., Traulsen, A., Nowak, M.A.: Active linking in evolutionary games. J. Theor. Biol. 243, 437-443 (2006)

24. Traulsen, A., Nowak, M.A., Pacheco, J.M.: Stochastic dynamics of invasion and fixation. Phys. Rev. E 74, 011909 (2006)

25. Santos, F.C., Rodrigues, J.F., Pacheco, J.M.: Epidemic spreading and cooperation dynamics on homogeneous small-world networks. Phys. Rev. E 72, 056128 (2005) 